\title{
Two-stage stochastic model on rattling vibration with amplitude modulation
}

\author{
Jian Ming Wen* and Qi Feng \\ Key Laboratory of Solid Mechanics of MOE, Tong-ji University, Si Pin Road 1329, Shanghai 200092, P.R. China
}

Received 24 April 2000

Revised 2004

\begin{abstract}
Rattling vibration in gear boxes of automobiles is an annoying problem. It may be generated in those gear wheels of a car transmission system, which are unloaded. In recent years, very general models have been developed to analyze the rattling phenomenon. One of them, in consideration of plays being the consequence of tolerances, backlashes and others, is modeled as an impulsive system that consists of some unloaded gears being able to rattle. Modern research has shown that the chaotic vibration may occur on a rattling system. In this paper, a stochastic modulated rattling system with two-stage was studied. A discrete stochastic model described by mean map was established using non-Gaussian technique. By the analysis of the example this model can reveal chaotic stochastic behaviors.
\end{abstract}

Keywords: Stochastic model, rattling vibration, non-Gaussian technique, random chaos, gear box

\section{Introduction}

Rattling vibration in gear boxes of automobiles is an annoying problem. It may be generated in those gear wheels of a car transmission system, which are not under load. In recent years, the rattling phenomenon has been received attention by many scientists and researchers [1-8]. The very general models have been developed to analyze the rattling phenomenon. One of them, in consideration of plays being the consequence of tolerances, backlashes and others, is modeled as an impulsive system that consists of some unloaded gears being able to rattle. Modern research has shown that the chaotic vibration can occur on a rattling system.

In 1988, Pfeiffer came up with an idea from Fermi's experiment and elaborated a discrete model using a map to describe the rattling vibration [4]. In 1990, Pfeiffer and Kunter introduced stochastic model into the analysis considering the effects of an additional noise [6]. The random model is also more realistic because a finite level of noise is present everywhere in reality. In 1998, Q.Feng and F. Pfeiffer proposed the discrete stochastic model describing the single rattling system by a mean map [7], and they revealed the stochastic vibration of rattling. The two-stage model describing the rattling vibration has been established by kunter [8]. However, his analysis was only limited to the investigation of deterministic chaos using the stochastic perturbing technique. In fact, due to influence of the noise, another type of vibration can be introduced. On the one hand, except for an additional noise, a random modulation of the control parameter of a nonlinear system can also induce interesting behavior [9]. On the other hand, chaotic stochastic vibration may occur in random dynamical systems [10]. In this paper, these problems were considered. A discrete stochastic model described by the mean map was established using non-Gaussian technique for the two-stage rattling system. By means of analysis of an example it is proved that the model can reveal the chaotic stochastic behavior. Through investigation, the Poincaré maps exhibit typical chaotic vibrations and the power spectrum of the mean velocity shows the characteristics of a continuous spectrum. The maximal exponent of Lyapunov showed the region of chaos by parameter $\alpha$. The findings in a special impulsive system are also significant for chaos study.

\footnotetext{
*Corresponding author: E-mail: wenjianming@mail.tongji.edu.cn.
} 


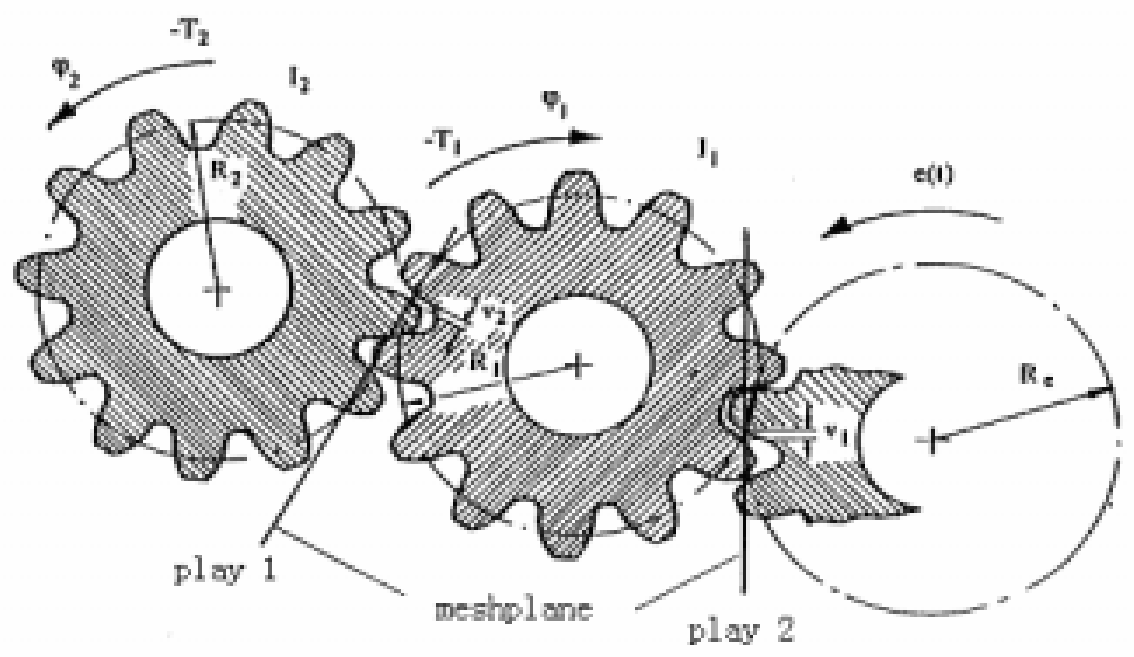

Fig. 1. Mechanical model.

\section{Mechanical model}

In order to establish the stochastic model, a brief review about the deterministic model is necessary. The deterministic model was put forward by Kunter in 1992 [8], in which a two-stage rattling system is considered. In Fig. 1, the driven gear wheel not under load can move freely between backlashes, and the free flight motion is only stopped by the backlash boundary, where an impact occurs. The motion of the driven wheel can be distinguished into two phases: the free flight phase and the contact phase. In the free flight phase, the equation of motion can be written as [8]:

$$
I_{i} \ddot{\varphi}_{i}+d_{i} \dot{\varphi}_{i}=-T_{i} \quad(i=1,2)
$$

In the contact phase, it can be expressed by

$$
\left(\begin{array}{c}
\dot{\varphi}_{1}^{+} \\
\dot{\varphi}_{2}^{+}
\end{array}\right)=\frac{1}{I_{1} R_{2}^{2}+I_{2} R_{1}^{2}} \cdot\left(\begin{array}{cc}
I_{1} R_{2}^{2}-\varepsilon I_{2} R_{1}^{2} & (1+\varepsilon) I_{2} R_{1} R_{2} \\
(1+\varepsilon) I_{1} R_{1} R_{2} & I_{2} R_{1}^{2}-\varepsilon I_{1} R_{2}^{2}
\end{array}\right)\left(\begin{array}{c}
\dot{\varphi}_{1}^{-} \\
\dot{\varphi}_{2}^{-}
\end{array}\right)
$$

In which $i(i=1,2)$ is the number of gear wheels; $R_{1}$ and $R_{2}$ are basic radius; $\varphi_{1}$ and $\varphi_{2}$ stand for the angular displacements; $I_{1}$ and $I_{2}$ denote the inertia moments of driven wheels; $d_{1}$ and $d_{2}$ are mean damping ratios in the free flight phase; $T_{1}$ and $T_{2}$ are constant moments; $\varepsilon$ is the restitution coefficient. The signs (-) and (+) denote short time before and after impact; $e(t)$ is a harmonic rotation with amplitude $a$ and $\omega$ frequency;

The above motion equation can be reformed to describe by relative displacement and relative velocity. If

$$
\begin{aligned}
& x_{1}=\frac{R_{e} e(t)-R_{1} \varphi_{1}}{\nu_{1}}, x_{2}=\frac{R_{2} \varphi_{2}-R_{1} \varphi_{1}}{\nu_{2}}, \tau=\omega t, \\
& \beta_{i}=\frac{d_{i}}{I_{i} \omega}, i=1,2, \gamma_{i}=\frac{T_{i} R_{i}}{I_{i} \nu_{i} \omega^{2}}, i=1,2,
\end{aligned}
$$

the following dimensionless equation are obtained

$$
\left\{\begin{array}{l}
\dot{x}_{1}=y_{1} \\
\dot{y}_{1}=-\beta_{1} y_{1}+\gamma_{1}+\ddot{f}+\beta_{1} \dot{f} \\
\dot{x}_{2}=y_{2} \\
\dot{y}_{2}=-\beta_{1} y_{2}+\left(\beta_{2}-\beta_{1}\right) y_{1}+\gamma_{1}-\gamma_{2}+\left(\beta_{1}-\beta_{2}\right) \dot{f}
\end{array}\right.
$$

in the free flight phase: $x_{1} \in(0.5,0.5), x_{2} \in\left(-\frac{\nu_{2}}{2 \nu_{1}}, \frac{\nu_{2}}{2 \nu_{1}}\right)$, and 


$$
y_{1}^{+}=y_{1}^{-}-\frac{I_{2} R_{1}^{2}(1+\varepsilon)}{I_{1} R_{2}^{2}+I_{2} R_{1}^{2}} y_{2}^{-}, \quad y_{2}^{+}=-\varepsilon y_{2}^{-}
$$

in the contact phase: $x_{1} \in\{-0.5,0.5\}, x_{2} \in\left\{-\frac{\nu_{1}}{2 \nu_{2}}, \frac{\nu_{1}}{2 \nu_{2}}\right\}$, where $x_{i},(i=1,2)$ and $y_{i},(i=1,2)$ denote relative displacement and relative velocity, $\nu_{i},(i=1,2)$ are plays respectively. $\beta_{i},(i=1,2)$ is the damping ratio in the free flight phase, $\gamma_{i},(i=1,2)$ indicates constant moment. $f=\alpha \sin \tau, \alpha=\frac{R_{e} a}{\nu_{1}}, \tau$ is dimensionless time. $\alpha$ stands for excitation amplitude. An accurate solution by a map for the above deterministic equations has been derived [8].

\section{Discrete stochastic model}

In deterministic model, the plays between gear teeth are assumed constant which is not fitting for the reality, because even with modern production possibilities no gear tooth can be manufactured in an ideal way and there is a lot of additional small irregularities due to the complete system behavior. It is assumed that the magnitude of backlash consists of two parts: average values and measure tolerance. After normalization, the first backlash magnitude is equal to $1+2 c_{1}$, and the second backlash magnitude is $\frac{\nu_{1}}{\nu_{2}}+2 c_{2}$. Then a modified equation of motion can be written as:

$$
\begin{aligned}
& \text { in free flight phase: } x_{1 s} \in\left(-\left(0.5+c_{1}\right),\left(0.5+c_{1}\right)\right), x_{2 s} \in\left(-\left(\frac{\nu_{1}}{2 \nu_{2}}+c_{2}\right),\left(\frac{\nu_{1}}{2 \nu_{2}}+c_{2}\right)\right) \\
& \qquad\left\{\begin{array}{l}
\dot{x}_{1 s}=y_{1 s} \\
\dot{y}_{1 s}=-\beta_{1} y_{1 s}+\gamma_{1}+(\alpha+\sigma \eta(\tau))\left(-\sin \tau+\beta_{1} \cos \tau\right) \\
\dot{x}_{2 s}=y_{2 s} \\
\dot{y}_{2 s}=-\beta_{1} y_{2 s}+\left(\beta_{2}-\beta_{1}\right) y_{1 s}+\left(\gamma_{1}-\gamma_{2}\right)+\left(\beta_{1}-\beta_{2}\right)(\alpha+\sigma \eta(\tau)) \cos \tau
\end{array}\right.
\end{aligned}
$$

Where, the subscript $s$ indicates the perturbed system that differs from unperturbed systems Eqs (3) and (4), $x_{i s}, y_{i s},(i=1,2)$ stand for relative displacement and relative velocity of the perturbed system, $\alpha$ is the deterministic part of the excitation amplitude. $\sigma$ represents the intensity of random forces described by a standard Gaussian white noise $\eta(\tau)$. That satisfied the following conditions.

$$
E[\eta(\tau)]=0 \quad E[\eta(\tau) \eta(s)]=\delta(\tau-s)
$$

In the contact phase: $x_{1 s} \in\left\{-\left(0.5+c_{1}\right),\left(0.5+c_{1}\right)\right\}, x_{2 s} \in\left\{-\left(\frac{\nu_{1}}{2 \nu_{2}}+c_{2}\right),\left(\frac{\nu_{1}}{2 \nu_{2}}+c_{2}\right)\right\}$, the modified equation of motion has the form of

$$
y_{1 s}^{+}=y_{1 s}^{-}-\frac{I_{2} R_{1}^{2}(1+\varepsilon)}{I_{1} R_{2}^{2}+I_{2} R_{1}^{2}} y_{2 s}^{-} \quad y_{2 s}^{+}=-\varepsilon y_{2 s}^{-}
$$

In general, when noise intensity is small, the stochastic model Eqs (5) and (6) can be considered as a modification for the deterministic models Eqs (3) and (4). Equation (5) is a nonlinear equation without a high order term that can be decoupled. In order to derive a discrete stochastic model, it is attempted to decouple the Eqs (5) and (6) into two parts: deterministic and stochastic. It is assumed that the solution of perturbed systems Eqs (5) and (6) can be separated into deterministic and stochastic parts with the form:

$$
\begin{aligned}
& x_{1 s}=x_{1}+\xi_{1}, \quad y_{1 s}=y_{1}+\zeta_{1}, \\
& x_{2 s}=x_{2}+\xi_{2}, \quad y_{2 s}=y_{2}+\zeta_{2}
\end{aligned}
$$

In which, $x_{i},(i=1,2)$ and $y_{i},(i=1,2)$ are the solutions of unperturbed system Eqs (3) and (4), while $\xi_{i}$ and $\zeta_{i}$ are stochastic variables. Substituting Eq. (7) into Eqs (5) and (6) and subtracting Eqs (3) and (4), we can obtain the motion equation of the stochastic part as following.

In the free flight phase:

$$
\xi_{1} \in\left(-\left(0.5+c_{1}-x_{1}\right),\left(0.5+c_{1}-x_{1}\right)\right) \xi_{2} \in\left(-\left(\frac{\nu_{1}}{2 \nu_{2}}+c_{2}-x_{2}\right),\left(\frac{\nu_{1}}{2 \nu_{2}}+c_{2}-x_{2}\right)\right)
$$




$$
\left\{\begin{array}{l}
\dot{\xi}_{1}=\zeta_{1} \\
\dot{\zeta}_{1}=-\beta_{1} \zeta_{1}+\sigma \eta(\tau)\left(-\sin \tau+\beta_{1} \cos \tau\right) \\
\dot{\xi}_{2}=\zeta_{2} \\
\dot{\zeta}_{2}=-\beta_{2} \zeta_{2}+\left(\beta_{2}-\beta_{1}\right) \zeta_{1}+\sigma \eta(\tau)\left(\beta_{1}-\beta_{2}\right) \cos \tau
\end{array}\right.
$$

In the contact phase:

$$
\begin{aligned}
& \xi_{1} \in\left\{-\left(0.5+c_{1}-x_{1}\right),\left(0.5+c_{1}-x_{1}\right)\right\} \xi_{2} \in\left\{-\left(\frac{\nu_{1}}{2 \nu_{2}}+c_{2}-x_{2}\right),\left(\frac{\nu_{1}}{2 \nu_{2}}+c_{2}-x_{2}\right)\right\} \\
& \zeta_{1}^{+}=\zeta_{1}^{-}-\frac{I_{2} R_{1}^{2}(1+\varepsilon)}{I_{1} R_{2}^{2}+I_{2} R_{1}^{2}} \zeta_{2}^{-} \xi_{2}^{+}=-\varepsilon \zeta_{2}^{-}
\end{aligned}
$$

Now Eqs (5) and (6) have been separated into two parts. The deterministic part is the same as Eqs (3) and (4). The stochastic part consists of Eqs (8) and (9). Integrating Eq. (3), if the sequence of the system states before or after impacts are known, $x_{i},(i=1,2)$ and $y_{i},(i=1,2)$ can be defined exactly. Considering the mapping before impact $H: X_{i, k}^{-} \rightarrow X_{i, k+1}^{-}$, in which $X_{i}=\left[x_{i}, y_{i}\right]^{T}$ is a state vector of the unperturbed system, an iterated operator is obtained as derived in reference [8].

$$
\begin{aligned}
x_{1, k+1}^{-}= & x_{1, k}^{-}+\left(-\varepsilon y_{1, k}^{-}-\alpha_{m} \cos \tau_{k}-\frac{\gamma_{1}}{\beta_{1}}\right) \cdot \frac{1}{\beta_{1}}\left(1-\exp \left(-\beta_{1} \Delta \tau_{k}\right)\right) \\
& +\alpha_{m}\left(\sin \tau_{k+1}-\sin \tau_{k}\right)+\frac{\gamma_{1}}{\beta_{1}} \Delta \tau_{k} \\
y_{1, k+1}^{-}= & \alpha_{m} \cos \tau_{k+1}+\frac{\gamma_{1}}{\beta_{1}}+\left(-\varepsilon y_{1, k}^{-}-\alpha_{m} \cos \tau_{k}-\frac{\gamma_{1}}{\beta_{1}}\right) \exp \left(-\beta_{1} \Delta \tau_{k}\right) \\
x_{2, k+1}^{-}= & x_{2, k}^{-}+\left(y_{2, k}^{-}-y_{1, k}^{-}+\alpha_{m} \cos \tau_{k}+\frac{\gamma_{2}}{\beta_{2}}\right) \cdot \frac{1}{\beta_{2}}\left(1-\exp \left(-\beta_{2} \Delta \tau_{k}\right)\right)+\left(\frac{\gamma_{1}}{\beta_{1}}-\frac{\gamma_{2}}{\beta_{2}}\right) \Delta \tau_{k} \\
& +\frac{1}{\beta_{1}}\left(-\varepsilon y_{1, k}^{-}-\alpha_{m} \cos \tau_{k}-\frac{\gamma_{1}}{\beta_{1}}\right) \cdot\left(1-\exp \left(1-\beta_{1} \Delta \tau_{k}\right)\right) \\
y_{2, k+1}^{-}= & \left(\frac{\gamma_{1}}{\beta_{1}}-\frac{\gamma_{2}}{\beta_{2}}\right)+\left(y_{2, k}^{-}-y_{1, k}^{-}+\alpha_{m} \cos \tau_{k}+\frac{\gamma_{2}}{\beta_{2}}\right) \exp \left(-\beta_{2} \Delta \tau_{k}\right) \\
& +\left(-\varepsilon y_{1, k}^{-}-\alpha_{m} \cos \tau_{k}-\frac{\gamma_{1}}{\beta_{1}}\right) \exp \left(-\beta_{1} \Delta \tau_{k}\right)
\end{aligned}
$$

In the above operator, the time difference $\Delta \tau_{k}$ of the two successive impacts has not been defined yet.

Equation (8) in the free flight phase is a non-linear stochastic differential equation without the restitution force term whose exact solution cannot be solved. The correlation coefficient $\rho$ of the random variables $\xi_{i}$ and $\zeta_{i}$ from the corresponding moment equation of Eq. (9) does not always satisfy the relation $\rho_{\xi_{i} \zeta_{i}} \leqslant 1$, therefore, its distribution is out of accord with a Gaussian normal distribution. Here, $\rho_{\xi_{i} \zeta_{i}}=\frac{C_{\xi_{i} \zeta_{i}}}{\sigma_{\xi_{i}} \sigma_{\zeta_{i}}}, C_{\xi_{i} \zeta_{i}}$ is covariance, $\sigma_{\xi_{i}}$ and $\sigma_{\zeta_{i}}$ are the standard derivations.

In order to derive a mean iterated operator, a non-Gaussian closure technique is used. An Edgeworth expansion is adopted to approximate the true distribution [11]. It is assumed that the variables $\xi_{1}$ and $\zeta_{1}$ have nothing to do with $\xi_{2}$ and $\zeta_{2}$ because the variables $\xi_{2}$ and $\zeta_{2}$ have not arisen in the equation about $\xi_{1}$ and $\zeta_{1}$. They follow the two-dimensional Edgeworth expansion respectively. The two-dimensional Edgeworth expansion is expressed as follow [11].

$$
p^{*}(\xi, \zeta)=P(\xi, \zeta)\left\{\sum_{n=0}^{N} \frac{\rho^{n}}{n !} H_{n}\left(\frac{\xi}{\sigma_{\xi}}\right) H_{n}\left(\frac{\zeta}{\sigma_{\zeta}}\right) \sum_{j+l=3}^{N} \frac{1}{j ! l !} \frac{\lambda_{j l}}{\sigma_{\xi}^{j} \sigma_{\zeta}^{1}} \sum_{n=0}^{N} H_{n+j}\left(\frac{\xi}{\sigma_{x i}}\right) H_{n+l}\left(\frac{\zeta}{\sigma_{\zeta}}\right)\right.
$$




$$
\begin{aligned}
& +\sum_{j+l=4}^{N} \frac{1}{j ! l !} \frac{\lambda_{j l}}{\sigma_{\xi}^{j} \sigma_{\zeta}^{1}} \sum_{n=0}^{N} \frac{\rho^{n}}{n !} H_{n+j}\left(\frac{\xi}{\sigma_{x i}}\right) H\left(\frac{\zeta}{\sigma_{\zeta}}\right) \frac{1}{2} \sum_{\substack{j+l=3 \\
r+s=4}}^{N} \frac{1}{j ! l ! r ! s !} \frac{\lambda_{j l}}{\sigma_{x i}^{j} \sigma_{\zeta}^{1}} \frac{\lambda_{r s}}{\sigma_{\xi}^{r} \sigma_{\zeta}^{s}} \\
& \left.\sum_{n=0}^{N} \frac{\rho^{n}}{n !} H_{n+j+r}\left(\frac{\xi}{\sigma_{\xi}}\right) H_{n+l+s}\left(\frac{\zeta}{\sigma_{\zeta}}\right)\right\}
\end{aligned}
$$

In which, $\lambda_{n p}=E\left[\xi^{n} \zeta^{p}\right](n=j$ or $r, p=l$ or $s)$ is moment, $\rho_{\xi \zeta}=\frac{\lambda_{11}}{\sigma_{\xi} \sigma_{\zeta}}$ is the correlation, $H_{k}(z)$ is the polynomial in the form of reference [11].

$$
H_{0}(z)=1, H_{1}(z)=z, H_{2}(z)=z^{2}-1, \ldots \ldots, H_{n+1}(z)=z H_{n}(z)-n H_{n-1}(z), \ldots \ldots
$$

Where $P(\xi, \zeta)$ means a standard Gaussian normal distribution, it has the form

$$
P(\xi, \zeta)=\frac{1}{2 \pi \sigma_{\xi} \sigma_{\zeta}} \exp \left(-\frac{\left(\xi-m_{\xi}\right)^{2}}{2 \sigma_{\xi}^{2}}\right) \exp \left(-\frac{\left(\zeta-m_{\zeta}\right)^{2}}{2 \sigma_{\zeta}^{2}}\right)
$$

where the standard derivations $\sigma_{i}(i=\xi, \zeta)$ and the mean values $m_{i}(i=\xi, \zeta)$ can be solved from the 2-order and the 1-order moment equation, respectively. According to the reference [7], the Eq. (11) can be deduced for the state before impact.

$$
P_{k+1}^{*-}(\xi, \zeta)=P(\xi, \zeta) \sum_{i=0}^{N} \sum_{j=0}^{N}\left[a_{i j}\right]_{k+1}^{-} H_{i}\left(\frac{\xi}{\sigma_{\xi}}\right) H_{j}\left(\frac{\zeta}{\sigma_{\zeta}}\right)
$$

Where the coefficients $\left[a_{i j}\right]_{k+1}^{-}$are the function related to the moments $\left[\lambda_{n p}\right]_{k+1}^{-}$and can be expressed as:

$$
\begin{gathered}
{\left[a_{00}\right]_{k+1}^{-}=1 ; \quad\left[a_{10}\right]_{k+1}^{-}=0 ; \quad\left[a_{20}\right]_{k+1}^{-}=0 ; \quad\left[a_{30}\right]_{k+1}^{-}=\frac{\left[\lambda_{30}\right]_{k+1}^{-}}{6 \sigma_{\xi}^{3}} ;} \\
{\left[a_{01}\right]_{k+1}^{-}=0 ; \quad\left[a_{21}\right]_{k+1}^{-}=\frac{\left[\lambda_{21}\right]_{k+1}^{-}}{2 \sigma_{\xi}^{2} \sigma_{\zeta}} ; \quad\left[a_{11}\right]_{k+1}^{-}=\frac{\left[\lambda_{11}\right]_{k+1}^{-}}{\sigma_{\xi} \sigma_{\zeta}}=\rho_{k+1}^{-} ;}
\end{gathered}
$$

In our problem, the moment $m_{n p}$ about $\xi_{2}$ and $\zeta_{2}$ can be determined as follows: $\dot{m}_{n p}=\frac{\partial E\left\lfloor\psi_{n p}\right\rfloor}{\partial \tau}$

$$
\dot{m}_{n p}=\frac{\partial E\left\lfloor\psi_{n p}\right\rfloor}{\partial \tau}=E\left[\zeta_{2} \frac{\partial \psi_{n p}}{\partial \xi_{2}}\right]-\beta_{2} E\left[\zeta_{2} \frac{\partial \psi_{n p}}{\partial \zeta_{2}}\right]+\left(\beta_{2}-\beta_{1}\right) E\left[\zeta_{1}\right] E\left[\frac{\partial \psi_{n p}}{\partial \zeta_{2}}\right]+\frac{\sigma^{2}}{2} E\left[\frac{\partial^{2} \psi_{n p}}{\partial \zeta_{2}^{2}}\right]
$$

in the free flight phase, where $n, p=1,2,3, \ldots, \psi_{n p}=\xi_{2}^{n} \zeta_{2}^{p}$. In the contact phase, there exists the impact constrained conditions of the moments as:

$$
\left\{\begin{array}{ll}
m_{n p}^{+}=-\varepsilon m_{n p}^{-}, & p \neq 0 \\
m_{n p}^{+}=m_{n p}^{-}, & p=0
\end{array} \quad(n, p=0,1,2, \ldots)\right.
$$

The moment $\lambda_{n p}$ about $\xi_{1}$ and $\zeta_{1}$ can be satisfied the following equation:

$$
\dot{\lambda}_{n p}=\frac{\partial E\left\lfloor\varphi_{n p}\right\rfloor}{\partial \tau}=E\left[\zeta_{1} \frac{\partial \varphi_{n p}}{\partial \xi_{1}}\right]-\beta_{1} E\left[\zeta_{1} \frac{\partial \varphi_{n p}}{\partial \zeta_{1}}\right]+\frac{\sigma_{0}^{2}}{2} \sin ^{2}\left(\tau+\alpha_{0}\right) E\left[\frac{\partial^{2} \varphi_{n p}}{\partial \zeta_{1}^{2}}\right]
$$

in the free flight phase. In which, $\varphi_{n p}=\xi^{n} \zeta^{p}, \sigma_{0}=\sigma \sqrt{1+\beta_{1}^{2}}, \sin \alpha_{0}=\frac{\beta_{1}}{\sqrt{1+\beta_{1}^{2}}}, \cos \alpha_{0}=\frac{-1}{\sqrt{1+\beta_{1}^{2}}}$. In the contact phase, there exist the impact-constrained conditions of the moments as: 


$$
\left\{\begin{array}{l}
\lambda_{n p}^{+}=\lambda_{n p}^{-}-\frac{I_{2} R_{1}^{2}(1+\varepsilon)}{I_{1} R_{2}^{2}+I_{2} R_{1}^{2}} m_{n p}^{-}, p \neq 0 \\
\lambda_{n p}^{+}=\lambda_{n p}^{-}, p=0
\end{array},(n, p=0,1,2, \ldots)\right.
$$

In the free flight phase, the moment equation chains Eq. (14) are closed and the moments can be solved uniquely without closing tolerance. Therefore the approximately distribution $P^{*}(\xi, \zeta)$ may be tend to be true if $N \rightarrow \infty$ [11].

According Eqs (14), (15), (16) and (17), all moments in the time interval $\Delta \tau_{k}$ can be obtained when the initial conditions $\left[\lambda_{n p}\right]_{0}=0$ and $\left[m_{n p}\right]_{0}=0$ are used. Based on the Eq. (13), the probability distribution of system between the two successive impacts can be approached by Eqs (18) and (19).

$$
\begin{aligned}
& p_{1, k+1}^{*-}\left(\xi_{1}, \zeta_{1}\right)=P\left(\xi_{1}, \zeta_{1}\right) \sum_{i=0}^{N} \sum_{j=0}^{N}\left[a_{i j}\right]_{k+1}^{-} H_{i}\left(\frac{\xi_{1}}{\sigma_{\xi_{1}}}\right) H_{j}\left(\frac{\zeta_{1}}{\sigma_{\zeta_{1}}}\right) \\
& p_{2, k+1}^{*-}\left(\xi_{2}, \zeta_{2}\right)=P\left(\xi_{2}, \zeta_{2}\right) \sum_{i=0}^{N} \sum_{j=0}^{N}\left[b_{i j}\right]_{k+1}^{-} H_{i}\left(\frac{\xi_{2}}{\sigma_{\xi_{2}}}\right) H_{j}\left(\frac{\zeta_{2}}{\sigma_{\zeta_{2}}}\right)
\end{aligned}
$$

After twice integration of Eq. (18), the discrete mean values of random variables $\xi_{1}$ and $\zeta_{1}$ for the state before impact are expressed as follows.

$$
\begin{aligned}
& E\left[\xi_{1}\right]_{k+1}^{-}=\int_{d_{11}}^{d_{12}} \xi_{1} \int_{-\infty}^{+\infty} P_{1, k+1}^{*-}\left(\xi_{1}, \zeta_{1}\right) d \zeta_{1} d \xi_{1}=\sigma_{\xi_{1}} A_{k+1}^{-} \\
& E\left[\zeta_{1}\right]_{k+1}^{-}=\int_{-\infty}^{+\infty} \zeta_{1} \int_{d_{11}}^{d_{12}} P_{1, k+1}^{*-}\left(\xi_{1}, \zeta_{1}\right) d \xi_{1} d \zeta_{1}=\sigma_{\zeta_{1}} B_{k+1}^{-}
\end{aligned}
$$

Where

$$
\begin{aligned}
d_{11}= & -\left(0.5+c_{1 \max }+x_{1}\right), d_{12}=0.5+c_{2 \max }-x_{1} \\
A_{k+1}^{-}= & \psi_{0}+\left[a_{40}\right]_{k+1}^{-}\left(\psi_{4}-2 \psi_{2}+\psi_{0}\right)+\ldots \ldots \\
\psi_{i}= & \left(\frac{d_{11}}{\sigma_{x i_{1}}}\right)^{i} \exp \left(-\frac{d_{11}^{2}}{2 \sigma_{\xi_{1}}^{2}}\right)-\left(\frac{d_{12}}{\sigma \xi_{1}}\right) \exp \left(-\frac{d_{12}^{2}}{2 \sigma_{\xi_{1}}^{2}}\right) B_{k+1}^{-}=\sum_{i=1}^{N}\left[a_{11}\right]_{k+1}^{-} \cdot m_{i} \\
m_{1}= & \psi_{0} ; m_{2}=\psi_{1} ; \\
& \ldots \ldots \\
m_{3}= & \psi_{2}-\psi_{0} ; m_{4}=\psi_{3}-3 \psi_{1} ;
\end{aligned}
$$

Using the same way, the discrete mean variable of random variables $\xi_{2}$ and $\zeta_{2}$ can be obtained.

$$
\begin{aligned}
& E\left[\xi_{2}\right]_{k+1}^{-}=\int_{d_{21}}^{d_{22}} \xi_{2} \int_{-\infty}^{+\infty} P_{2, k+1}^{*-}\left(\xi_{2}, \zeta_{2}\right) d \zeta_{2} d \xi_{2}=\sigma_{\xi_{2}} C_{k+1}^{-} \\
& E\left[\zeta_{2}\right]_{k+1}^{-}=\int_{-\infty}^{+\infty} \xi_{2} \int_{d_{21}}^{d_{22}} P_{2, k+1}^{*-}\left(\xi_{2}, \zeta_{2}\right) d \xi_{2} d \zeta_{2}=\sigma_{\xi_{2}} D_{k+1}^{-}
\end{aligned}
$$

Where

$$
\begin{aligned}
d_{21} & =-\left(\frac{\nu_{1}}{2 \nu_{2}}+c_{2 \max }+x_{2}\right) ; \quad d_{22}=\frac{\nu_{1}}{2 \nu_{2}}+c_{2 \max }-x_{2} \\
C_{k+1}^{-} & =\varphi_{0}+\left[b_{30}\right]_{k+1}^{-} \varphi_{3}+\left[b_{40}\right]_{k+1}^{-}\left(\varphi_{4}-2 \varphi_{2}+\varphi_{0}\right)+\ldots \ldots \\
\varphi_{i} & =\left(\frac{d_{21}}{\sigma_{\xi_{2}}}\right)^{i} \exp \left(-\frac{d_{21}^{2}}{2 \sigma_{\xi_{2}}}\right)-\left(\frac{d_{22}}{\sigma_{\xi_{2}}}\right)^{i} \exp \left(-\frac{d_{22}^{2}}{2 \sigma_{\xi_{2}}}\right)
\end{aligned}
$$




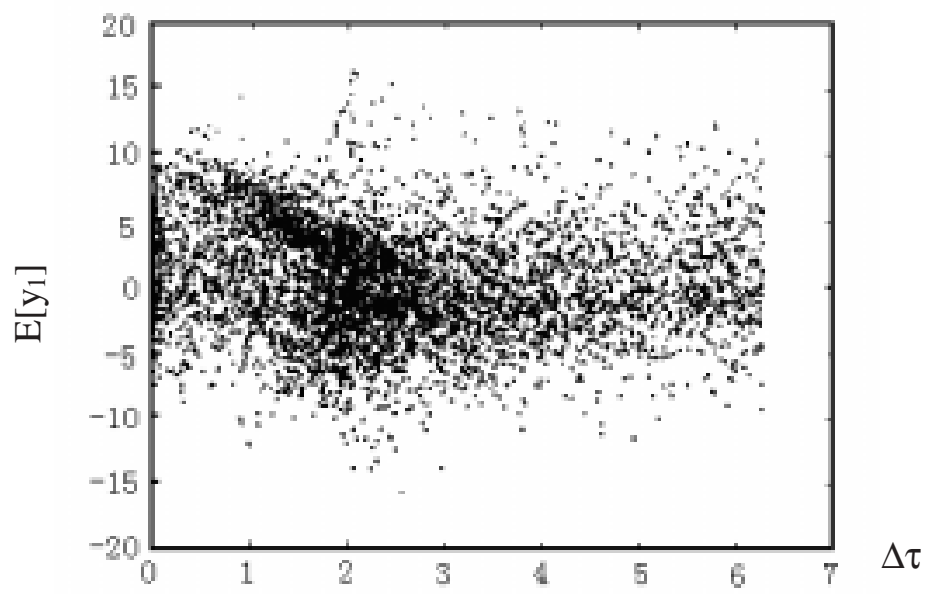

(a)

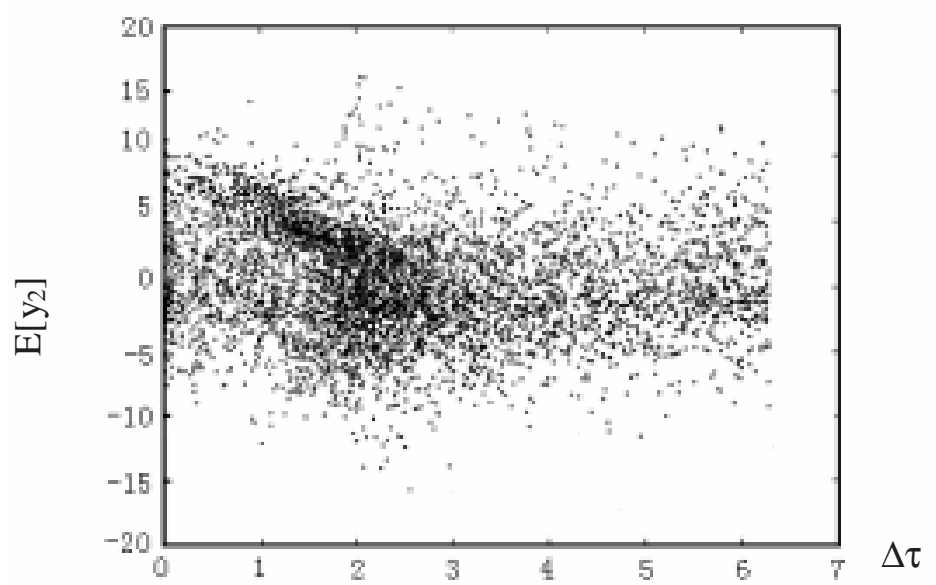

(b)

Fig. 2. the mean poincaré map. $E\left[y_{1}\right], E\left[y_{2}\right]$ - mean velocity; $\Delta \tau$-time difference

$$
\begin{aligned}
D_{k+1}^{-} & =\sum_{i=1}^{N}\left[b_{i 1}\right]_{k+1}^{-} \cdot k_{i} \\
k_{1} & =\varphi_{0} ; \quad k_{2}=\varphi_{1} ; \quad k_{3}=\varphi_{2}-\varphi_{0} ; \quad k_{4}=\varphi_{3}-3 \varphi_{1} ; \ldots \ldots
\end{aligned}
$$

If the iterated operator Eqs (7), (20) and (21) are superposed a mean map $H_{s}: E\left[X_{i s}\right]_{k}^{-} \rightarrow E\left[X_{i s}\right]_{k+1}^{-}$for the state before impact to describe the two-stage stochastic rattling system, five dimensional mean mapping can be derived as

$$
\begin{aligned}
E\left[x_{1 s}\right]_{k+1}^{-}= & x_{1, k+1}^{-}+\sigma_{\xi_{1}} A_{k+1}^{-} \\
E\left[y_{1 s}\right]_{k+1}^{-}= & \left(-\varepsilon E\left[y_{1 s}\right]_{k}^{-}-\alpha \cos \tau_{k}-\frac{\gamma_{1}}{\beta_{1}}\right) \cdot \exp \left(-\beta_{1} \Delta \tau_{k}\right)+\frac{\gamma_{1}}{\beta_{1}}+\alpha \cos \tau_{k+1} \\
& +\left(B_{k+1}^{-}+\varepsilon B_{k}^{-} \exp \left(-\beta_{1} \Delta \tau_{k}\right)\right) \cdot \sigma_{\zeta_{1}} \\
E\left[x_{2 s}\right]_{k+1}^{-}= & x_{2, k+1}^{-}+\sigma_{\xi_{2}} \cdot C_{k+1}^{-}
\end{aligned}
$$




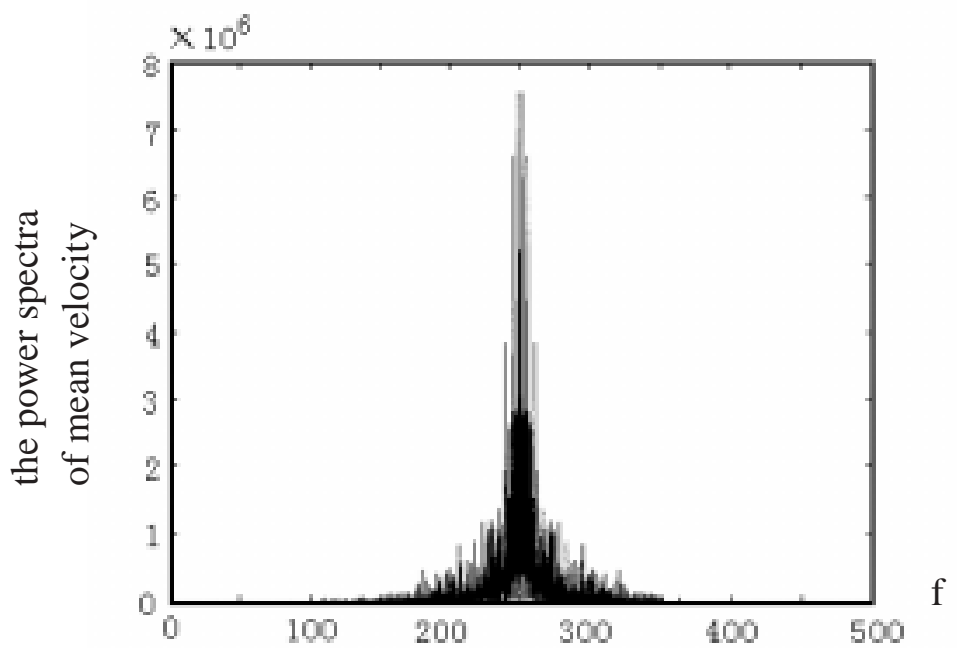

(a)

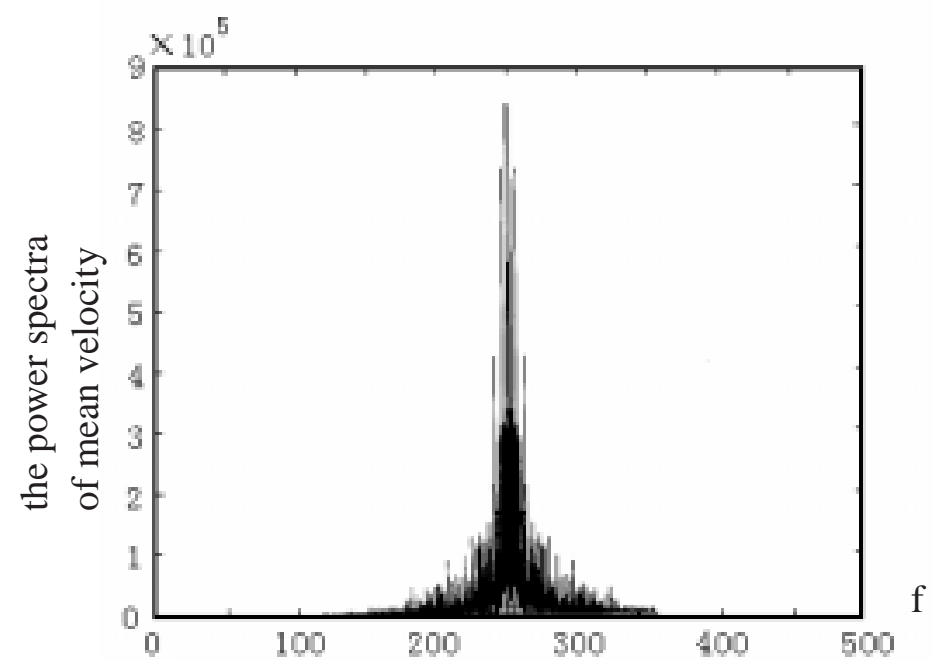

(b)

Fig. 3. the power spectra of mean velocity, $f-$ frequency.

$$
\begin{aligned}
E\left[y_{2 s}\right]_{k+1}^{-}= & \left(E\left[y_{2 s}\right]_{k}^{-}-(1+\varepsilon) E\left[y_{1 s}\right]_{k}^{-}-\alpha \cos \tau_{k}-\frac{\gamma_{1}}{\beta_{1}}\right) \exp \left(-\beta_{1} \Delta \tau_{k}\right) \\
& +\left(E\left[y_{2 s}\right]_{k}^{-}-E\left[y_{1 s}\right]_{k}^{-}+\alpha \cos \tau_{k}+\frac{\gamma_{2}}{\beta_{2}}\right) \cdot \exp \left(-\beta_{2} \Delta \tau_{k}\right)+\left(\frac{\gamma_{1}}{\beta_{1}}-\frac{\gamma_{2}}{\beta_{2}}\right) \\
& +\left(D_{k+1}^{-}-D_{k}^{-} \exp \left(-\beta_{2} \Delta \tau_{k}\right)-D_{k}^{-} \exp \left(-\beta_{2} \Delta \tau\right)\right) \sigma_{\zeta_{2}} \\
& +\left((1+\varepsilon) \exp \left(-\beta_{1} \Delta \tau_{k}\right)+\exp \left(-\beta_{2} \Delta \tau_{k}\right)\right) B_{k}^{-} \cdot \sigma_{\zeta_{1}} \\
\tau_{k+1}=\tau_{k}+ & \Delta \tau_{k}, \quad \Delta \tau_{k}=\min \left(\Delta \tau_{k, 1}, \Delta \tau_{k, 2}\right)(\bmod u l o, 2 \pi)
\end{aligned}
$$

Now the time difference $\Delta \tau_{k}$ of the two successive impacts can be solved from the following mesh condition of the gears: $E\left[x_{i s}\right]_{k+1}^{-}=-E\left[x_{i s}\right]_{k}^{-}$. 


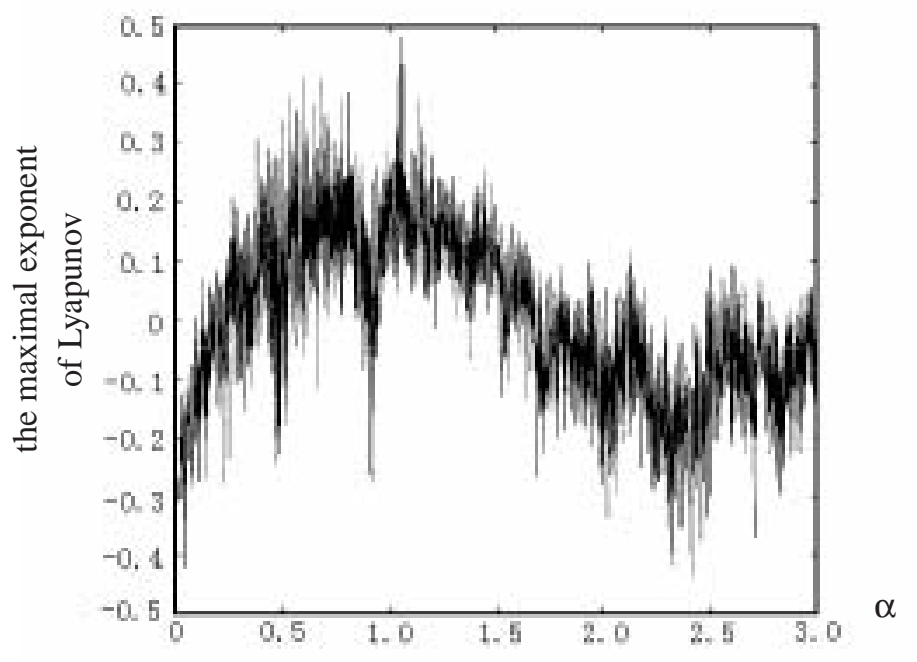

Fig. 4. the maximal exponent of Lyapunov.

\section{Analysis of example}

In order to study the above model, data from the reference [8] is used as follows:

DAT21 [8]: $\alpha=1.5, \quad \varepsilon=0.9, \omega=300 \mathrm{~s}^{-1}$,

$I_{i}=4000.0 \mathrm{kgmm}^{2}, I_{2}=2500 \mathrm{kgmm}^{2}, R_{1}=41.0 \mathrm{~mm}, R_{2}=26.4 \mathrm{~mm}$,

$\nu_{1}=\nu_{2}=0.2 \mathrm{~mm}, d_{1}=d_{2}=100 \mathrm{Nmms}, T_{1}=100 \mathrm{Nmm}, T_{2}=50 \mathrm{Nmm}$.

Performing the iterated operator Eq. (22) for the above data and the noise intensity $\sigma=\sqrt{\alpha}$ and the initial condition $Y_{s, 0}=\{2.0,2.0\}$ and $X_{s 0}=\{0.5,0.5\}, d_{m}=0.5$ and considering counter meshes of the gears $E\left[x_{i s}\right]_{k+1}=-E\left[x_{i s}\right]_{k}$, the representative calculating results are shown in the following figures. Figure 2(a,b) are mean Poincaroo map, in which the horizontal coordinate is the time difference $\Delta \tau_{k}$, and the vertical coordinate is the mean velocity before impact $E\left[y_{i s}\right]_{k+1}^{-}$. The structures of Fig. 2 display a fuzzy set of points. Figure 3(a,b) show the power spectra of mean velocity, in which the horizontal coordinate is the dimensionless frequency $f$, and the vertical coordinate is the power spectral density of mean velocity. In the Fig. 3 narrow band spectra are exhibited. Through Figs 2 and 3 the above model may reveal chaotic stochastic behavior. Figure 4 indicates the maximal exponent of Lyapunov, in which the horizontal coordinate is the parameter $\alpha$ and the vertical coordinate is the maximal exponent of Lyapunov. Figure 4 shows the region of chaos by parameter $\alpha$. From the Fig. 4 , it can be obtained that the maximal exponent of Lyapunov is plus when $0.2 \leqslant \alpha \leqslant 1.7$. In this range, the chaos can be happen.

\section{Conclusions}

In the present paper, a discrete stochastic model has been developed to describe two-stage rattling vibration in gearbox. It is well known that rattling vibration cause noise and its deterministic model is only an ideal one, so that in the present work the use of a stochastic model described by a mean map is suggested to investigation rattling noise. In our study, the mean Poincaré map, the power spectrum density and the maximal exponent of Lyapunov have been investigated for the dynamics of random chaos. The calculation results have shown that the mean Poincaroo maps exhibit chaos and the power spectra of mean velocity display the behavior of a continuous spectrum. The result of the maximal exponent of Lyapunov has shown that the chaotic stochastic vibration can be controlled by variation of a two-stage rattling system parameter. Comparing the two-stage model with the single stage model [7], both models reveal the random chaos on the dynamics of a rattling system. The existe the random chaos in the rattling system has been justified again. 


\section{References}

[1] F. Kücükay, Dynamik der Zahnradgetriebe, Modell, Verfahren, Verhalten, Springer Verlag, Berling-Heidelberg, 1987.

[2] F. Kücükay and F. Pfeiffer, Über Rassel in kfz-Schaltgetrieben, Ing-Archiv 56 (1986), 25-37.

[3] F. Pfeiffer, On steady dynamics in machines with plays, Proceedings of the 7th world congress on the theory of machines and mechanisms, Sevilla, 1987

[4] F. Pfeiffer Seltsame Attraktoren in Zahnradgetrieben, Ing-Archiv 58 (1988), 113-125.

[5] K. Karagiannis and F. Pfeiffer Theoretical and experimental investigations of gear-rattling, Nonlinear Dynamics 2 (1991), $367-387$.

[6] F. Pfeiffer and A. Kunert, Rattling model from deterministic to stochastic Processes, Nonlinear Dynamics 1 (1990), 63-74.

[7] Q. Feng and F. Pfeiffer, Stochastic model on a rattling system, J. Sound Vib 215(3) (1998), 439-453.

[8] A. Kunter, Dynamik spielbehafteter Maschinenteile, Springer Verlag, Berling-Heidelberg, 1992.

[9] F. Moss and P.V.E. McVlintock, Noise in nonlinear dynamical systems, (Vol. 2), Cambridge: Cambridge University Press, 1985

[10] T. Kapitamiak, Chaos in System with Noise, World Scientific, Singapore, 1988.

[11] R.A. Ibrahim, Parametric random vibration, John Willey and Sons INC, New York, 1985.

[12] C.F. Moon, Chaotic vibration, John Wiely and Sons, New York, 1987. 

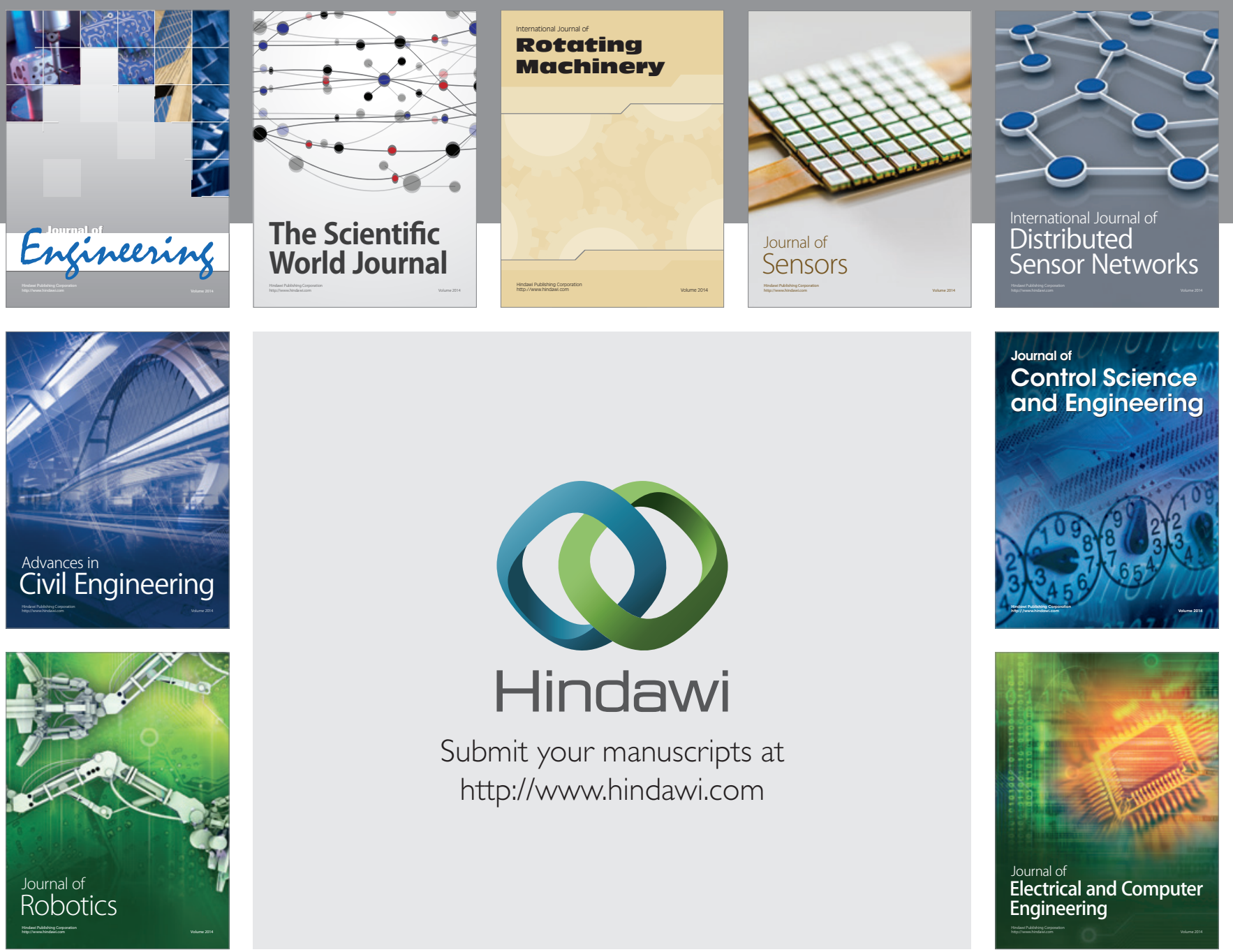

Submit your manuscripts at

http://www.hindawi.com
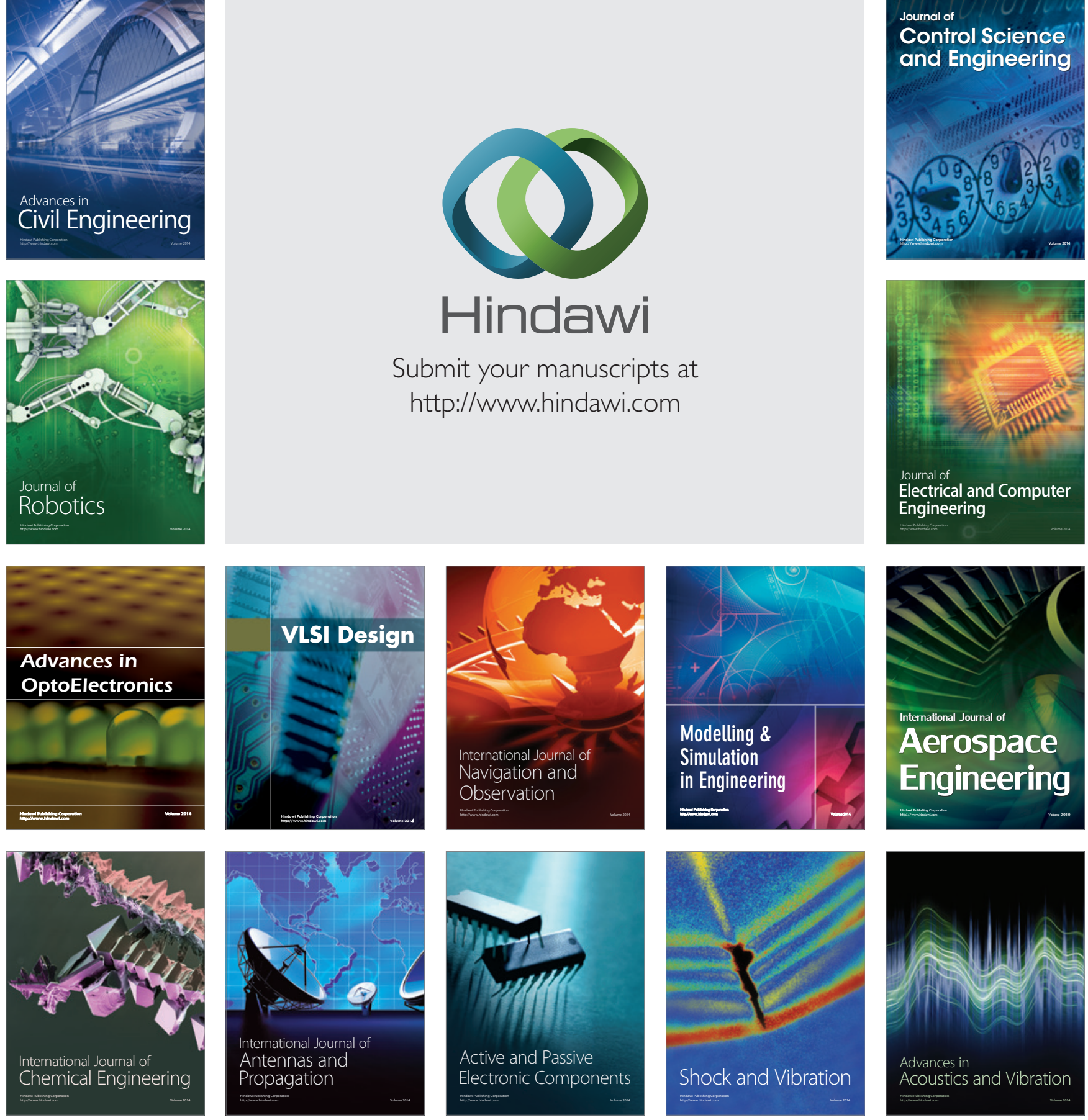